INPLASY

PROTOCOL

To cite: Peng et al. High

Tourniquet Pressure versus

Low Touniquet Pressure in

Total Knee Arthroplasty: A

systematic review and meta-

analysis. Inplasy protocol

2021110048. doi:

10.37766/inplasy2021.11.0048

Received: 15 November 2021

Published: 15 November 2021

Corresponding author:

Peng Yang

yangpeng2@stu.scu.edu.cn

Author Affiliation:

Westchina hospital of sichuan university.

Support: No financial support.

Review Stage at time of this submission: Data extraction.

Conflicts of interest:

None declared.

\section{High Tourniquet Pressure versus Low Touniquet Pressure in Total Knee Arthroplasty: A systematic review and meta-analysis}

\author{
Peng, Y1; Bin, S².
}

Review question / Objective: The objective of this study was to assess the efficacy and safety of high tourniquet pressure (HTP) for patients after total knee arthroplasty (TKA) compared with low tourniquet pressure (LTP).

Condition being studied: Tourniquet use is common in total knee arthroplasty. Many factors have influence on the development of post-tourniquet complications like tourniquet pressure, time, width, and type, while the pressure remains a critical element.However, no standard protocols yet have been established for the use of this device.

Information sources: PubMed, Embase, Web of Science, Cochrane and Ovid database were searched for studies published from inception of the databases to September 10st, 2021. Additionally, relevant journals and references of studies was hand-searched.

INPLASY registration number: This protocol was registered with the International Platform of Registered Systematic Review and Meta-Analysis Protocols (INPLASY) on 15 November 2021 and was last updated on 15 November 2021 (registration number INPLASY2021110048).

\section{INTRODUCTION}

Review question / Objective: The objective of this study was to assess the efficacy and safety of high tourniquet pressure (HTP) for patients after total knee arthroplasty (TKA) compared with low tourniquet pressure (LTP).
Condition being studied: Tourniquet use is common in total knee arthroplasty.Many factors have influence on the development of post-tourniquet complications like tourniquet pressure, time, width, and type, while the pressure remains a critical element.However, no standard protocols 
yet have been established for the use of this device.

\section{METHODS}

Search strategy: The study protocol, conducted according to the Cochrane standard."tourniquet pressure" and "knee replacement, knee arthroplasty and total knee arthroplasty" were used as key words in connection with AND or OR.

Participant or population: Patients undergoing total knee arthroplasty with tourniquet.

Intervention: Low tourniquet pressure (LTP) group involves cuff pressure not above $250 \mathrm{mmHg}$ or systolic blood pressure (SBP) plus $120 \mathrm{mmHg}$.

Comparator: High tourniquet pressure (HTP) group involves cuff pressure not less than $300 \mathrm{mmHg}$ or SBP plus $150 \mathrm{mmHg}$.

Study designs to be included: Randomised controlled trails

Eligibility criteria: (1) The study had to be a randomized controlled trail. (2) The intervention(s) evaluated in the trials had to be two or more different tourniquet pressures in primary TKAs and the results of both designs had to be reported separately.

Information sources: PubMed, Embase, Web of Science, Cochrane and Ovid database were searched for studies published from inception of the databases to September 10st, 2021. Additionally, relevant journals and references of studies was hand-searched.

Main outcome(s): Complications; pain; calculated blood loss; duration of surgery; hemoglobin (HB) drop; transfusion and range of motion (ROM).

Quality assessment / Risk of bias analysis: The risk of bias about the included studies was assessed by consulting the Cochrane Handbook (including random sequence generation, allocation concealment, blinding of participants and personnel, blinding of outcome assessment, incomplete outcome data, selective reporting, and other bias).

Strategy of data synthesis: Heterogeneity was evaluated using the $\mathbf{I} 2$ test.

Subgroup analysis: Sensitivity analyses and subgroup analyses were conducted to investigate possible sources of heterogeneity and the stability of the results.

Sensitivity analysis: Sensitivity analyses and subgroup analyses were conducted to investigate possible sources of heterogeneity and the stability of the results.

Language: English.

Country(ies) involved: China.

Keywords: tourniquet; pressure; total knee arthroplasty; pain; complication.

Contributions of each author:

Author 1 - Peng Yang.

Email: yangpeng_9686@163.com

Author 2 - Bin Shen.

Email: shenbin_1971@163.com 